\title{
Design for Mechanical Structure of Asynchronous Motor Principle Experiment Device
}

\author{
Zhongyao $\mathrm{Wu}^{1, \mathrm{a}}$, Jianzhong $\mathrm{Fu}^{2}$, Huilin $\mathrm{Qu}^{3}$, Jianbo $\mathrm{CaO}^{4, \mathrm{~b}}$ \\ ${ }^{1}$ College of Engineering, Zhejiang University, Hangzhou, Zhejiang 310027 \\ ${ }^{2}$ College of Engineering, Zhejiang University, Hangzhou, Zhejiang 310027 \\ ${ }^{3}$ College of Engineering, Zhejiang University, Hangzhou, Zhejiang 310027 \\ ${ }^{4}$ College of Engineering, Zhejiang Normal University, Jinhua, Zhejiang 321004 \\ a1553541784@qq.com, bcaojianbo2008@163.com
}

Keywords: Asynchronous motor, Experimental device, Optimization design

\begin{abstract}
To solve the expression difficulties of the basic principle of asynchronous motor, and the faults to understand the relevant knowledge not fully, the asynchronous motor intuitive to express the basic principle of the experiment device was designed by analyzing the existing mechanical structures of asynchronous motor. The three-dimensional modeling was finished for the mechanical structure of the asynchronous motor principle experiment device. The experimental device is mainly divided into the base part design, speed display design and the basic principle of rotating demonstration part design. Through asynchronous motor principle experiment device can verify and demonstrate the rotation principle of the asynchronous motor, thereby facilitating scholars to understand.
\end{abstract}

\section{Introduction}

With the continuous improvement of people's living standards and rapid development of economy, the application field of motors keeps expanding, and the market demand becomes huger and huger. Whether a nation is economically or culturally advanced can be measured by the average number of motors each family owns in this nation. Relevant data shows that the production of small motors reached seven billion in the year 2001, and it has been predicted that the coming years will continue to see an increase in the market demand of motors[1]. Although asynchronous motors are closely tied to daily life, as they have been applied in appliances such as electric fans, washing machines, refrigerators, pistol electric drills and so on, the teaching of asynchronous motors at school is far from practical [2]. In most classrooms, teachers teach theories and theories only. A lack of pragmatic content makes it almost impossible for students to bear in mind the relevant knowledge of asynchronous motors.

Asynchronous motor principle experiment device, consisting of the base part design, speed display design and the basic principle of rotating demonstration part design, is able to clearly display how asynchronous motors generate electricity. Though simple in design, it is effective and practical, and operates with a low cost and zero pollution.

\section{Mechanical Structure Design of the Asynchronous Motor Principle experimental Device}

The mechanical structure of the asynchronous motor principle experiment equipment mainly includes base part design, speed display design and the basic principle of rotating demonstration part design. The experimental platform adopts the under -device platform. The convex platform on the right side of the basal plane is installed with small DC motor 6 and connecting on horseshoe magnet 9 by the coupling. In the right bottom of pedestal open a slot 5 used to install the battery. Applying the basic principle of squirrel cage motor, the horseshoe magnet 9 and squirrel cage rotor 12 are fixed on the pedestal 1 . Through the rotation of the magnet cutting the magnetic induction line produces inductive electromotive force and induced current. Among them tachometer 2, switch 
button 3, turn button 4 play roles of speed adjusting, switch controlling and turning respectively. Through these two instruments indicating 8 and 11, a tachometer displays DC motor speed under the mediation of the circuit, and the other tachometer is used to display the rotor speed of the principle experiment.

Getting the 2D assembly drawing of asynchronous motor principle experiment device through the analysis and calculation, as it shown in Fig.1, 2 and 3. Among them 1 - base, 2 - tachometer, 3 switch button, 4 - turning button, 5 - the inside groove install batteries, 6 - small DC motors, 7 motor fixed seat, 8 - speed display table, 9 - support bar, 10 - horseshoe magnet.

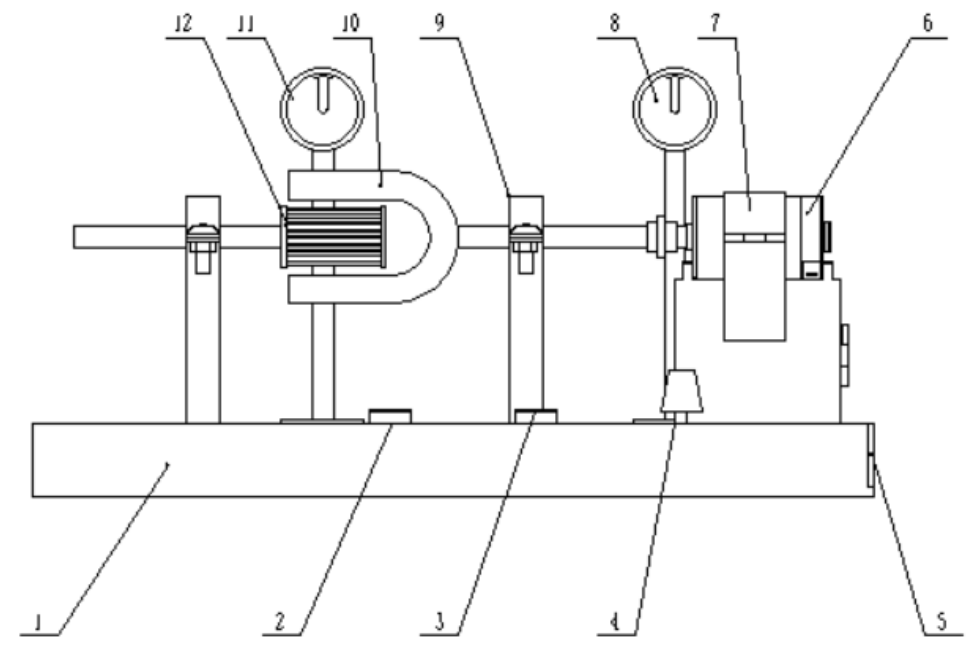

Fig. 1 Front view

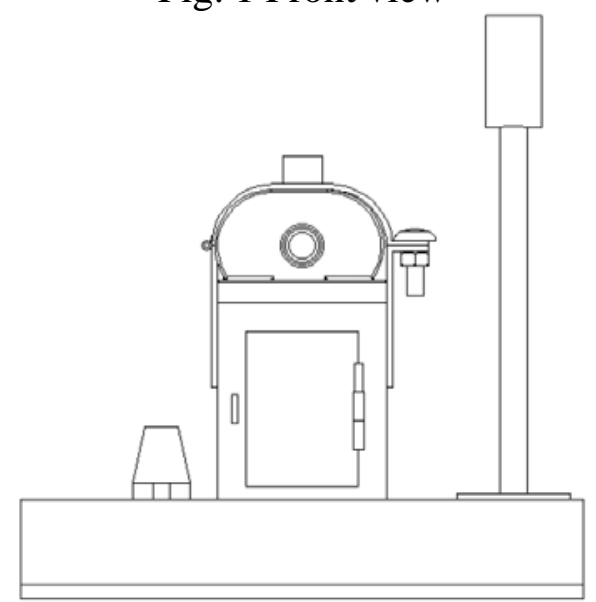

Fig. 2 Left view

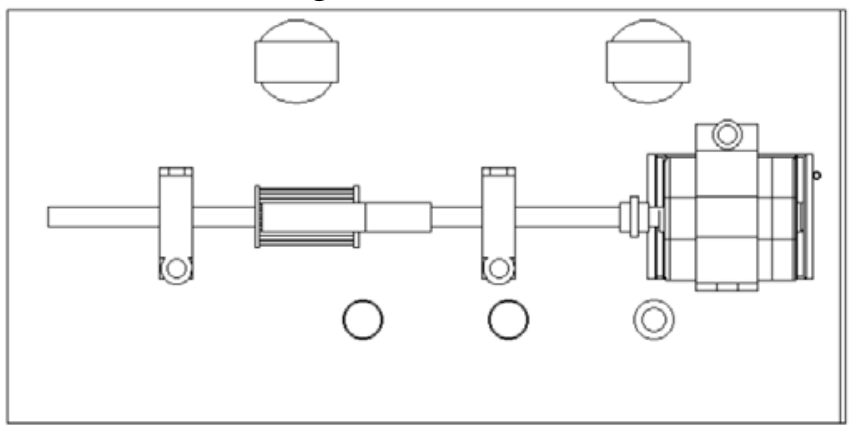

Fig. 3 Planform

\section{Control System Design of Asynchronous Motor Principle Experiment Device}

Small DC motor, one type of motors applied widely now, is directly installed into the circuit in the device, making the motor driver energy saving, highly efficient, miniaturization and lightening. Control system design in this paper is based on single chip microcomputer control DC motor controller, with functions of adjusting speed and positive inversion, increasing the reliability of the 
controller.

\section{Motor H Bridge Driving Circuit}

Transistor is divided into bipolar and MOS. Integrated circuit consists of them. For the sake of design, easy-using, reliability, the two kinds of transistors are made into a circuit.

It is important to ensure the two triodes on the same side of $\mathrm{H}$ Bridge are not conduction at the same time when driving the motor, otherwise the current will reach the maximum value (the current is only restricted by the power performance), even burning triode. Based on these improved circuits, we can increase four and-gates and two not-gates on the basis of the basic H Bridge circuit.

Applying these methods above, motor running only needs to use three control signals: two direction signals and an enable signal. If DIR-L signal is 0 , DIR-R signal is 1 , and enable signal is 1 , then the transistor Q1 and transistor Q4 conduct, the motor current from left to right (Fig. 4); If DIR-L signal turns into 1 and DIR-R signal changes to zero, then the Q2 and Q3 will be conducted, current reversed flowing through the motor.

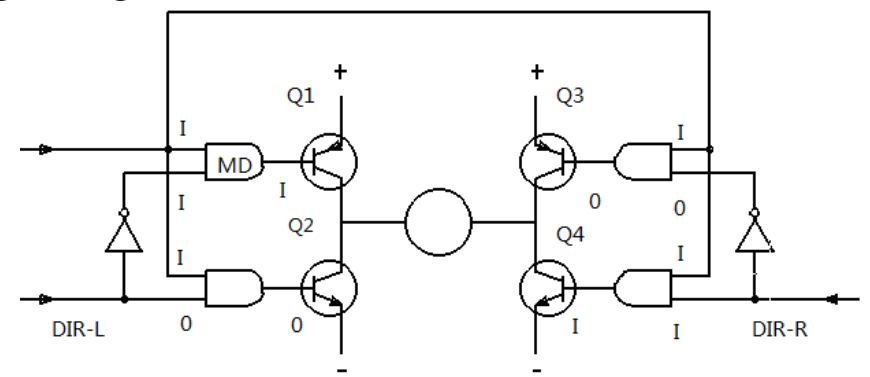

Fig. 4 H Bridge driving circuit of DC motor and using signals and direction of use

\section{The Basic Principle of PWM Control}

PWM (pulse width modulation) is a voltage adjustment method which controls the frequency of the DC power supply switch to change the voltage across the load, reaching the control request. In the adjustment system, PWM drive control connects and disconnects power supply according to a fixed frequency and according to the need to change the time of 'on' and 'off'. It changes the size of the average voltage by changing the DC motor armature voltage on the 'duty cycle', to control the speed of the motor. As the description above, PWM is known as 'switch drive' [3-6].

\section{Control Circuit Diagram Design of the Principle Experiment Device}

Control system design based on controller of the single chip DC motor, it has positive and negative rotation function, increasing the reliability of the controller. Through the analysis above, the control circuit of the whole principle experiment device is shown in fig.5 below.

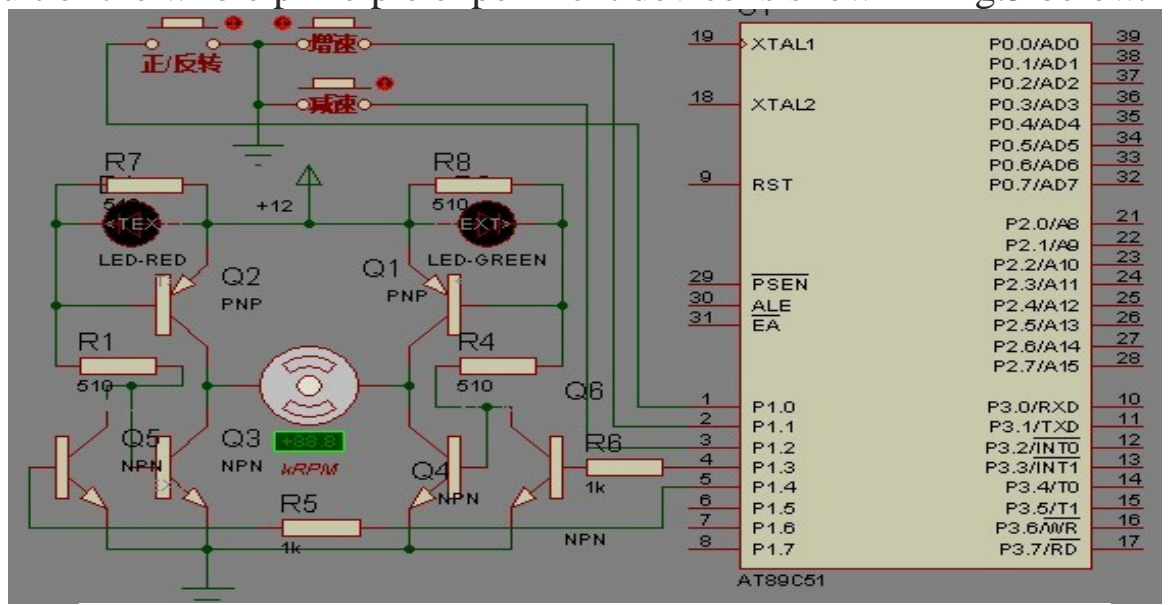

Fig. 5 control circuit diagram of principle experiment device 


\section{Conclusion}

This research puts forward a new design for the mechanical structure size of asynchronous motor principle experiment device with its assembly drawing drawn with the Solidworks software. This new device, simple but practical, is both low-cost and pollution-free.

\section{Acknowledgement}

The work was supported by the Experimental Technology Development Project of Zhe-jiang No rmal University (No. sj201416).

\section{References}

[1] Hailin Yuan. Development trends and prospects of small motor- impression of 2002 Visit to Japan [J]. Industry information,2002.3(2):32-36.

[2] Jianguo Ruan. The current situation and measures of the Motor efficiency [J]. Mechanical and electrical engineering,2009.6(5)1-8.

[3] N.Smith (S),Trent Polytechnic,An electronic load controller for waterpower edinduction generators[J].Iee review,1989.7:123-125.

[4] G.A.Shone*,RCCreppe,CRSou,The asynchronous motorone-dimension alan alysis[J]. The State University of Campinas, Brazil, 2009(10): 34-35.

[5] Zeming $\mathrm{Wu}$. The software realization method of generation of the PWM signal microcontroller[J]. Electronic technology, 2008 (01):20-21.

[6] Su Wang. Dc motor PWM speed control research and the realization of singlechip microcomputer control[J]. Mechanical and electrical engineering. 2008(11):82-110. 